\title{
c-myc but not Hif-1 $\alpha$-dependent downregulation of VEGF influences the proliferation and differentiation of HL-60 cells induced by ATRA
}

\author{
GUANHUA SONG ${ }^{1}$, YANMEI LI ${ }^{1}$, ZHIYONG ZHANG $^{1}$, XIA REN $^{1}$, HONGJIANG LI $^{1}$, WEN ZHANG ${ }^{1}$, \\ RUOYING WEI ${ }^{1}$, SUFEI PAN ${ }^{1}$, LULU SHI ${ }^{1}$, KEHONG BI ${ }^{2}$ and GUOSHENG JIANG ${ }^{1}$
}

\begin{abstract}
${ }^{1}$ Department of Hemato-Oncology, Institute of Basic Medicine, Shandong Academy of Medical Sciences, Key Laboratory for Modern Medicine and Technology of Shandong Province, Key Laboratory for Rare and Uncommon Diseases, Key Medical Laboratory for Tumor Immunology and Traditional Chinese Medicine Immunology of Shandong, Jinan, Shandong 250062; ${ }^{2}$ Qianfoshan Hospital of Shandong, Jinan, Shandong 250014, P.R. China
\end{abstract}

Received December 3, 2012; Accepted February 22, 2013

DOI: $10.3892 /$ or.2013.2395

\begin{abstract}
Vascular endothelial growth factor (VEGF) plays an important role in solid tumor growth, progression and metastasis as well as in the proliferation and differentiation of hematological malignancies. However, the molecular mechanism that modulates VEGF expression and secretion in leukemia cells has not yet to be elucidated. The purpose of the present study was to investigate the role of the signal pathway in modulating the expression of VEGF in HL-60 cells. Specific siRNAs targeting VEGF were transfected into HL-60 cells and the VEGF expression was measured with reverse transcription-polymerase chain reaction (RT-PCR) and western blot assay. The cell proliferation of HL-60 cells was detected by the cell counting kit- 8 (CCK-8) assay and the differentiation of HL-60 cells induced by all-trans-retinoic acid (ATRA) was detected by the RT-PCR assay and flow cytometry assay for CD11b. The upstream transcription factors that were related to VEGF expression such as P53, SP-1, c-jun, VHL, cox-2, c-myc and stat 3 were detected by RT-PCR assay. In addition, the chromatin immunoprecipitation (ChIP) assay was used to reveal the role of c-myc by binding the target gene VEGF. The results demonstrated the hypoxia-inducible factor $1 \alpha$-related signaling pathway, not the same as in solid tumors, might not play a key role in modulating VEGF expression. c-myc contributes to the modulation of VEGF expression by targeting the promoter of VEGF, which was indicated by the ChIP assay. In conclusion, our data demonstrate that VEGF
\end{abstract}

Correspondence to: Dr Kehong Bi, Qianfoshan Hospital of Shandong, 66 Jingshi Road, Jinan, Shandong 250014, P.R. China E-mail: kehongbi@126.com

Professor Guosheng Jiang, Department of Hemato-Oncology, Institute of Basic Medicine, Shandong Academy of Medical Sciences, 18877 Jingshi Road, Jinan, Shandong 250062, P.R. China

E-mail: jiangguosh@163.com

Key words: vascular endothelial growth factor, HL-60, c-Myc, differentiation, proliferation plays an important role in the differentiation and proliferation of HL-60 cells; c-myc-dependent downregulation of VEGF induced by ATRA contributes to the differentiation of HL-60 cells.

\section{Introduction}

The vascular endothelial growth factor (VEGF) family modulates several endothelial cell functions, particularly angiogenesis and lymphangiogenesis, which are involved in solid tumor growth, progression and metastasis (1). Higher expression of VEGF and levels of secretion have been demonstrated in human hematological malignancies. There is accumulating evidence that some leukemia cells secrete higher levels of VEGF and also express functional VEGF receptors (VEGFRs), such as VEGFR2 and VEGFR1, which result in the induction of an autocrine or paracrine loop. Previous findings demonstrated that high expression of VEGF can promote the proliferation and colony formation and inhibit the apoptosis of leukemic cells, which promotes the progression of hematopoietic tumors not only by stimulating vascular endothelial growth (2).

In general, the expression of VEGF is regulated by some intrinsic and extrinsic factors, with hypoxia and hypoglycemia being the major stimuli. Hypoxia-inducible factor $1 \alpha$ (HIF-1 $\alpha)$ has been demonstrated to be a key angiogenesis-triggering event, particularly in solid tumors. Previous studies have described that hypoxia can enhance invasion and metastasis in chronic myeloid leukemia (CML) (3), acute lymphatic leukemia (ALL) (4), chronic lymphatic leukemia (CLL) cells (5), possibly by activating VEGF.

Our previous study showed that the HIF- $1 \alpha$-related pathway was independent of the downregulation of VEGF in HL-60 cells induced by all-trans-retinoic acid (ATRA) (6). Furthermore, another study elucidated that HIF-1 $\alpha$ is an independent prognostic factor (7). Thus, a novel VEGF regulation system may exist in HL-60 cells.

VEGF plays a crucial pathogenic and prognostic role in acute myeloid leukemia (AML), including enhanced bone marrow vascularization (8). However, data concerning the 
biological function of VEGF and the molecular mechanism that modulates VEGF expression and secretion in leukemia cells, such as HL-60 cells, remain to be elucidated (9). The aim of the present study was to investigate the role of VEGF and the molecular mechanism for the transcriptional regulation of VEGF. We hypothesized that VEGF could trigger proliferation and inhibit differentiation for HL-60 cells, and some upstream key transcriptional factors may contribute to the modulation of VEGF expression by binding to its promoters.

\section{Materials and methods}

Materials. ATRA was purchased from Sigma-Aldrich Co., Ltd., and was dissolved in ethanol at $1 \mathrm{mM}$ and stored at -80 or $-20^{\circ} \mathrm{C}$. These stocks were diluted with the media to the desired concentrations immediately before the experiment, keeping the final concentration of ethanol at $0.1 \%$. All experiments were performed under low-light conditions to minimize retinoid photoisomerization. Anti-human VEGF antibody (19003-1-AP, ProteinTech Group, Wuhan, P.R. China), anti-human stat3 antibody (no. 9132, Cell Signaling Technology, USA), anti-human c-myc antibody (sc-42, Santa Cruz Biotechnology, Santa Cruz, CA) and anti-human CD11b antibody (11-0113, eBiosciences, San Diego, CA) were purchased. Cell counting kit-8 (CCK-8) was purchased from Dojindo Laboratories, Kumamoto, Japan.

Cell culture. The human promyelocytic leukemia cell line HL-60 was obtained from the Cell Bank of Type Culture Collection of the Chinese Academy of Sciences. HL-60 cells were cultured in IMDM medium (Hyclone, Logan, UT) supplemented with $20 \%$ fetal calf serum (FCS, Hyclone), incubated at $37^{\circ} \mathrm{C}, 5 \% \mathrm{CO}_{2}$. To prepare the cell model, HL-60 cells were seeded at $1 \times 10^{5} / \mathrm{ml}$ in $25 \mathrm{~cm}^{2}$ culture flasks in culture medium and supplemented with $1 \mu \mathrm{M}$ ATRA. Cells were harvested after treatment and used for the following experiments.

Differentiation assay. Control and HL-60 cells treated with ATRA $\left(1 \times 10^{6}\right.$ cells) were washed with PBS containing $1 \%$ FCS and $0.01 \%$ sodium azide were incubated for $30 \mathrm{~min}$ in FCS at $4^{\circ} \mathrm{C}$. Subsequently, FITC-conjugated anti-human CD11b antibody $(1 \mu \mathrm{g} / \mathrm{ml})$ was added to the cells and incubated at $25^{\circ} \mathrm{C}$ for $45 \mathrm{~min}$ followed by washing with PBS. The cells were then fixed in $1 \%$ paraformaldehyde and analyzed on Beckman Coulter Epics XL Flow cytometer. Isotypic rat IgG was also used to check for nonspecific binding.

Proliferation assay. HL-60 cells were treated withATRA $(1 \mu \mathrm{M})$. The cell proliferation was monitored at 24,48 and $72 \mathrm{~h}$ using the CCK- 8 assay according to the instructions, and the absorbance was read at $450 \mathrm{~nm}$ using a microplate enzyme-linked immunosorbent assay reader (Thermo Scientific Multiskan MK3).

Western blot assay. Thirty micrograms of protein extracts from HL-60 cells induced by ATRA or not were used for SDS-PAGE, and then transferred to PVDF membranes. The membranes were blocked with 5\% skimmed milk in TBST for $1 \mathrm{~h}$ and incubated with antibodies against VEGF, stat3 and c-myc at 1:1,000 dilutions overnight at $4^{\circ} \mathrm{C}$. After HRP-conjugated secondary antibody was added, proteins were detected using an ECL kit.
RNA isolation and reverse transcription-polymerase chain reaction (RT-PCR) assay. HL-60 cells grown to 50\% confluence were incubated either with or without ATRA at appropriate concentrations for three days. The cells were then lysed and their total RNAs were isolated using TRIzol (Invitrogen, USA) according to the manufacturer's instructions. For reverse transcription, samples were incubated in an Eppendorf PCR system at $42^{\circ} \mathrm{C}$ for $30 \mathrm{~min}$, then at $90^{\circ} \mathrm{C}$ for $5 \mathrm{~min}$ and at $5^{\circ} \mathrm{C}$ for $5 \mathrm{~min}$. Reverse transcription mixtures were subjected to PCR with specific primers. Primer sequences are shown in Table I. Reactions were incubated at $94^{\circ} \mathrm{C}$ for $2 \mathrm{~min}$ and were then amplified using temperature parameters of $94^{\circ} \mathrm{C}$ for $30 \mathrm{sec}$, $60^{\circ} \mathrm{C}$ for $30 \mathrm{sec}$, and $72^{\circ} \mathrm{C}$ for $30 \mathrm{sec}$. Amplifications were carried out for 35 cycles, followed by a $7-$ min extension at $72^{\circ} \mathrm{C}$. Results were normalized to GAPDH.

Specific siRNAs and transfection. siRNA oligonucleotides targeting stat3 (the target mRNA sequences, 5'-CACA TACAGGCTTAAGCTCTA-3') and c-myc (the target mRNA sequences, 5'-CTCGGTGCAGCCGTATTTCTA-3') were designed and synthesized by Qiagen along with HiPerFect transfection reagent. For the transfection assay with siRNA, $2 \times 10^{6}$ cells were grown to $80 \%$ confluence. The original stock of the siRNA was re-suspended in siRNA suspension $\mathrm{H}_{2} \mathrm{O}$ provided by the manufacturer. The resulting suspension was aliquoted in required amounts for each experiment and stored at $-20^{\circ} \mathrm{C}$ until it was ready to use. On the day of transfection, aliquots of siRNA suspension were diluted by culture medium without serum. The siRNA was then gently introduced into the cells by mixing with the required amount of HiPerFect transfection reagent at room temperature to allow formation of transfection complexes. The cells were harvested $48 \mathrm{~h}$ after transfection for analysis. Parallel experiments with $\mathrm{Mm} /$ Hs-MAPK1 and AllStar negative control provided with the kit were used as positive and negative controls in our experiment. Culture cells were harvested for western blotting and RT-PCR analysis.

Chromatin immunoprecipitation (ChIP) assay. ChIP assay was carried out in a standard manner, with minor modifications. HL-60 cells were treated with $1 \%$ formaldehyde for $15 \mathrm{~min}$. The cross-linked chromatin was then prepared and sonicated to an average size of 300-400 bp before being immunoprecipitated with antibodies specific to c-myc or control rabbit IgG at $4^{\circ} \mathrm{C}$ overnight. After reversal of cross-linking, the immunoprecipitated chromatin was amplified with polymerase chain reaction (PCR) and the resulting PCR products were separated by agarose gel electrophoresis.

Statistical analysis. Data are presented as the means \pm SE of three or four experiments. Analysis was performed using a Student's t-test. Values of $\mathrm{P}<0.05$ were considered to indicate a statistically significant difference.

\section{Results}

Effect of VEGF on differentiation and proliferation of HL-60 cells. In order to confirm the critical role of VEGF in the differentiation and proliferation of HL-60 cells, VEGF siRNA transfection was carried out in HL-60 cells. Treatment with 
Table I. Sequences of primers.

\begin{tabular}{lllll}
\hline Gene & Target & Species & \multicolumn{1}{c}{ Forward primer (5'-3') } & \multicolumn{1}{c}{ Reverse primer (5'-3') } \\
\hline GAPDH & mRNA & Human & ACATGTTCCAATATGATTCC & TGGACTCCACGACGTACTCAG \\
c-myc & mRNA & Human & GAAGTCATCCTGCCAGTCCC & CTGAAGTTTGCTGCACCGAC \\
stat3 & mRNA & Human & GGCTGGTAATTTATATAATCCCT & ACTAAAAGGCCAATACATTACAA \\
VEGF & mRNA & Human & CGGGAACCAGATCTCTCACC & AAAATGGCGAATCCAATTCC \\
SP-1 & mRNA & Human & AAGAAATGACCTTAGGAACATACCC & CCGTATATGTCTACACACAGATGAC \\
HIF-1 $\alpha$ & mRNA & Human & CCTATGTAGTTGTGGAAGTTTATGC & ACTAGGCAATTTTGCTAAGAATG \\
cox-2 & mRNA & Human & TTACAATGCTGACTATGGCTAC & CTGATGCGTGAAGTGCTG \\
c-jun & mRNA & Human & ACGACCTTCTATGACGATGCC & ATGTGCCCGTTGCTGGAC \\
VHL & mRNA & Human & TACCGAGGTCACCTTTGGC & GGAGGCATCGCTCTTTCAG \\
P53 & mRNA & Human & GTCCTGCTTGCTTACCTCGCTTAGT & ACCTGATTTCCTTACTGCCTCTTGC
\end{tabular}

VEGF, vascular endothelial growth factor; HIF-1 $\alpha$, hypoxia-inducible factor $1 \alpha$; VHL, von Hippel-Lindau syndrome.
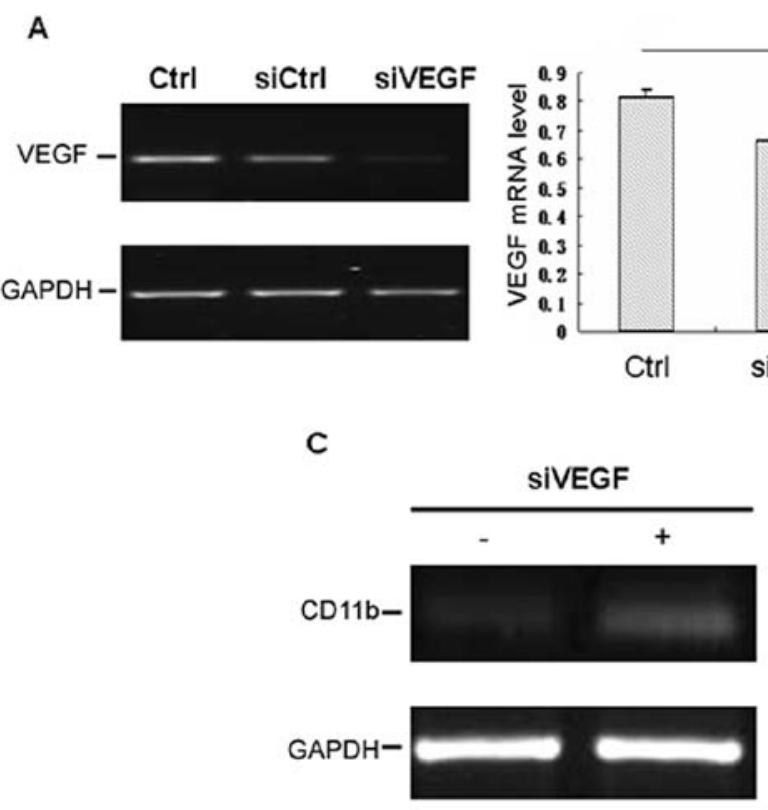
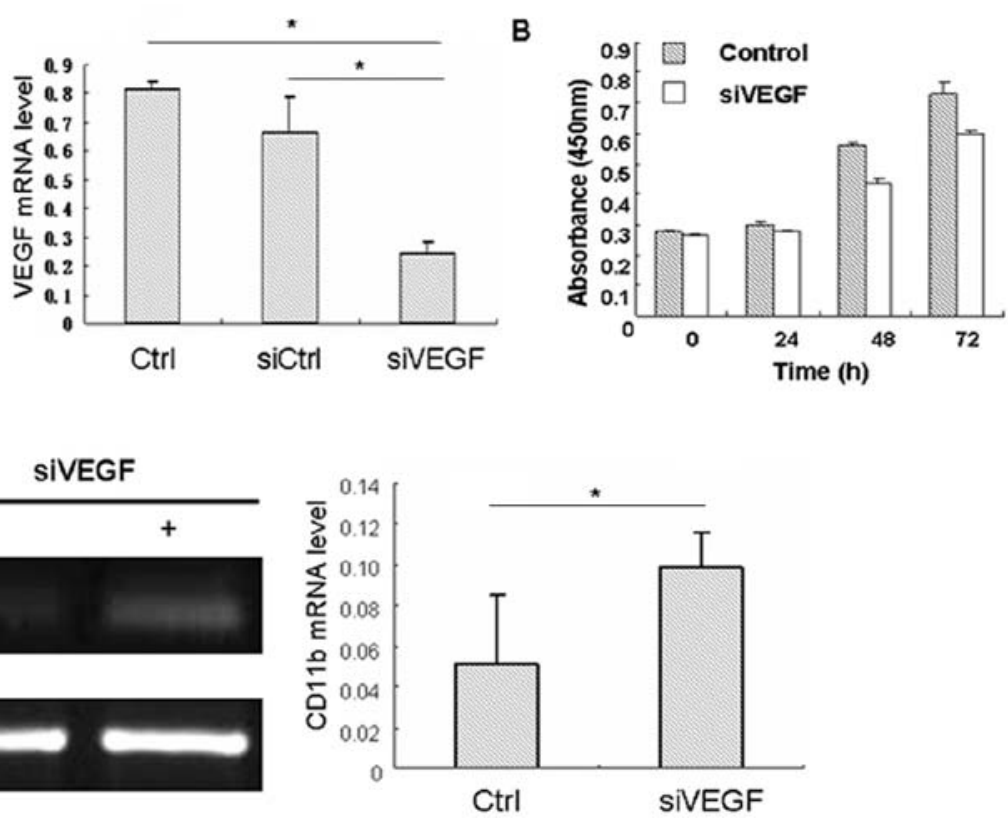

Figure 1. Effect of VEGF on differentiation and proliferation in HL-60 cells. (A) VEGF was silenced with VEGF siRNA by 78.2\%, compared with negative/ normal controls. (B) Classical differentiation marker CD11b was enhanced by reverse transcription-polymerase chain reaction (RT-PCR) in the VEGF siRNA group. (C) After VEGF was inhibited by siRNA, proliferation of HL-60 cells was inhibited via CCK- 8 assay at $450 \mathrm{~nm}$. The data shown were the means \pm SD of triplicate experiments. ${ }^{*} \mathrm{P}<0.05$.

VEGF siRNA reduced the production of VEGF by $78.2 \%$, as compared with normal controls (Fig. 1A). In order to detect the effect of VEGF on the proliferation of HL-60 cells, the proliferation was detected by CCK- 8 assay $48 \mathrm{~h}$ after transfection, and revealed that siRNA knockdown of VEGF could inhibit the proliferation of HL-60 cells as compared with that of control cells (Fig. 1B). Furthermore, the role of VEGF in the regulation of differentiation of HL-60 cells was also investigated, and the results indicated that the CD11b expression and positive percent was higher in the VEGF-siRNA group than in that of various controls (Fig. 1C), which suggested that HL-60 cells presented evidence of differentiation by silencing the VEGF expression. These data indicated that VEGF may play an important role in the progression of proliferation and differentiation in HL-60 cells.
Induction of differentiation and inhibition of proliferation of HL-60 cells by ATRA. Under experimental conditions of this study, $1 \mu \mathrm{M}$ ATRA induced differentiation of HL-60 cells, manifested by inhibition of cell proliferation, morphological changes and expression of differentiation marker CD11b. Cell proliferation was strongly inhibited following ATRA treatment (Fig. 2A). Also, morphological features of granulocytic differentiation, such as segmented nuclei and condensed chromatin, were clearly evident in ATRA-treated HL-60 cells (Fig. 2B). Moreover, the percentage of cells expressing CD11b was higher following ATRA treatment, as shown in Fig. 2C.

Downregulation of VEGF by ATRA is Hif-1 $\alpha$-independent in HL-60 cells. As previously reported, $1 \mu \mathrm{M}$ concentration of ATRA inhibited the growth of leukemia cells and down- 
A

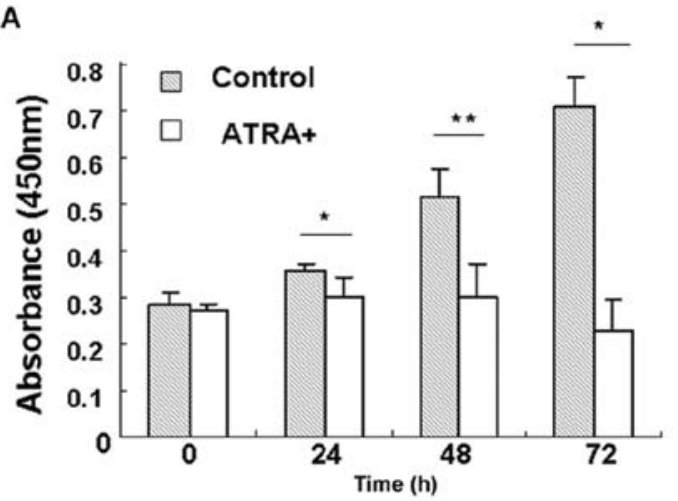

B

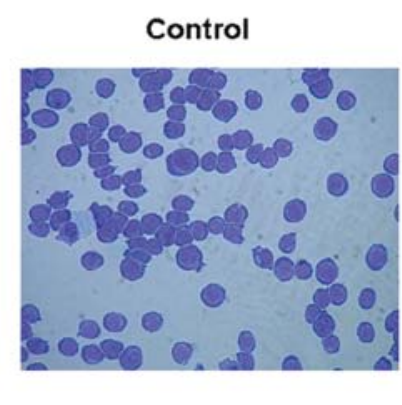

ATRA+

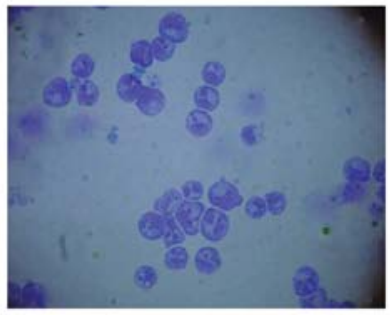

C
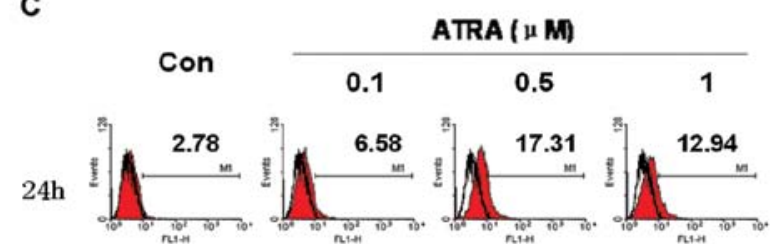

$48 \mathrm{~h} \int_{10^{\circ}}^{2.77}$
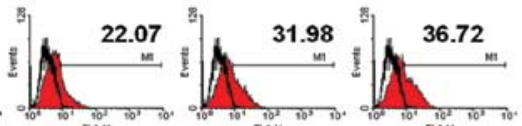

$72 \mathrm{~h} \underbrace{6.96}_{10.06}$
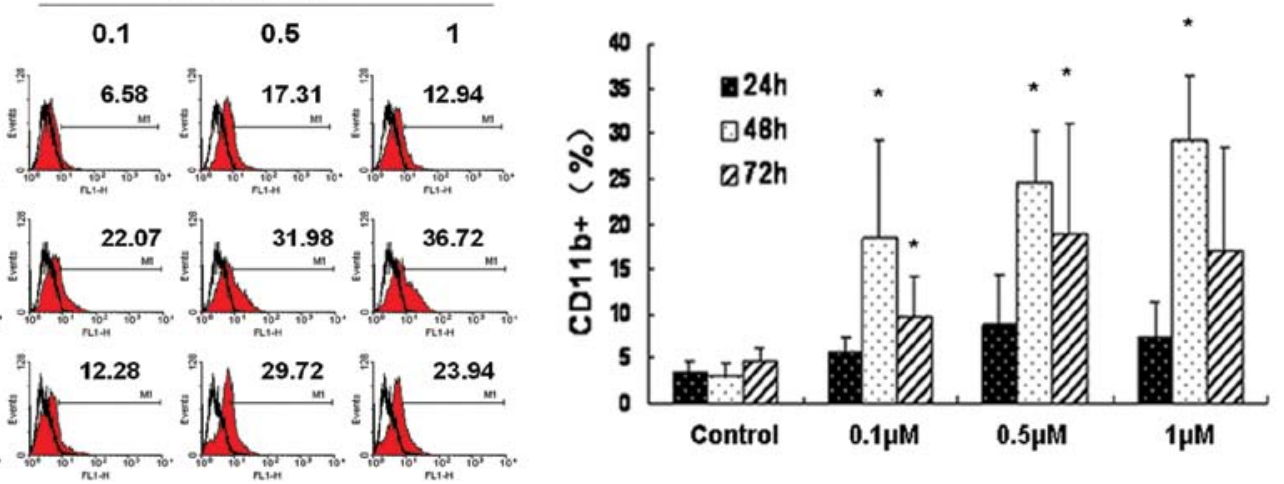

Figure 2. ATRA induces differentiation and inhibits proliferation of HL-60 cells. (A) Following ATRA treatment, cell proliferation was strongly inhibited by CCK-8 assay at $450 \mathrm{~nm}$. (B) Giemsa staining confirmed segmented nuclei and condensed chromatin with ATRA stimuli. (C) Flow cytometry analyzed expression of CD1 $1 \mathrm{~b}$ at $0.1,0.5,1 \mu \mathrm{M}$ and at $24,48,72 \mathrm{~h}$, respectively. CD1 lb was enhanced significantly as shown in the histogram. The data are presented as the means $\pm \mathrm{SE} .{ }^{*} \mathrm{P}<0.05 ;{ }^{* *} \mathrm{P}<0.01$ vs. control.

A

\begin{tabular}{ccc}
\multicolumn{3}{c}{ ATRA } \\
\hline 0 & 48 & $72 \mathrm{~h}$
\end{tabular}
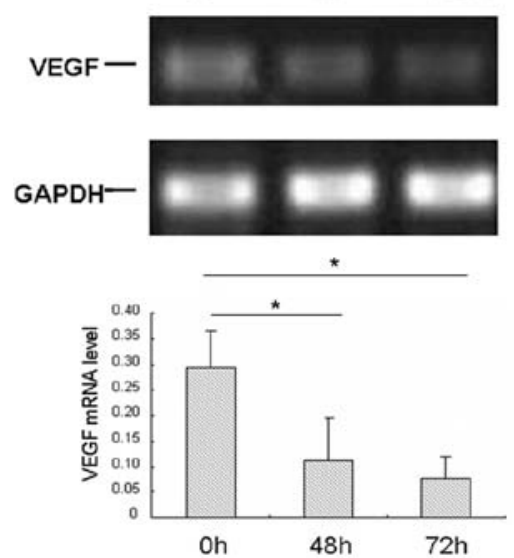

B

B
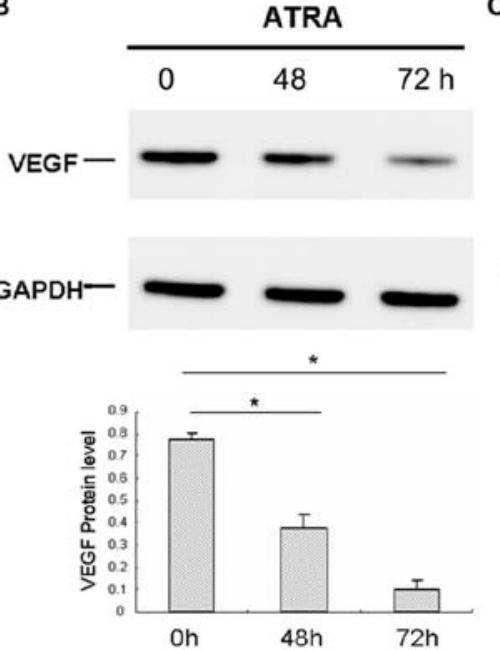

C
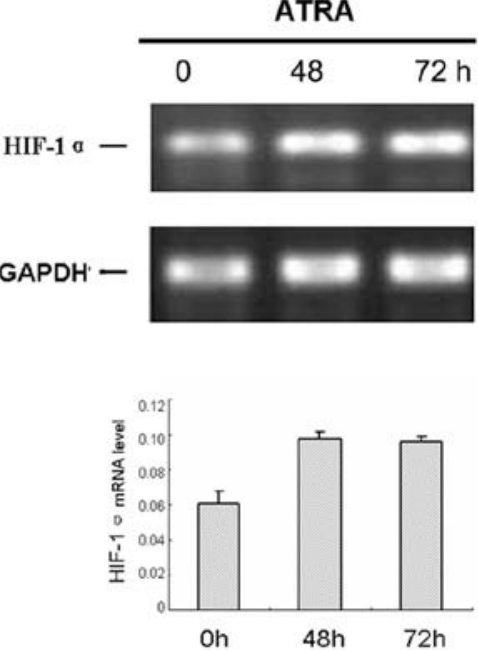

Figure 3. Downregulation of VEGF in HL-60 cells by ATRA is Hif-1 $\alpha$-independent. (A and B) Following ATRA stimulation for 48 and 72 h, VEGF mRNA and protein were decreased by RT-PCR and western blot analysis. (C) Hif- $1 \alpha$ mRNA was upregulated in HL-60 cells after exposure to ATRA for 48 and $72 \mathrm{~h}$ by RT-PCR analysis. ${ }^{*} \mathrm{P}<0.05$.

regulated the expression of VEGF (6). The mRNA and protein levels of VEGF were detected by RT-PCR and western blot assay, respectively, in HL-60 cells treated with ATRA. As indicated in Fig. 3A and B, ATRA downregulated the expression of VEGF in a time-dependent manner. The mRNA and protein levels of VEGF in HL-60 cells were decreased signifi- cantly in response to treatment with $1 \mu \mathrm{M}$ ATRA compared with the control. The housekeeping gene GAPDH was used for normalization. Hif-1 $\alpha$ mRNA was upregulated in HL-60 cells after exposure to ATRA, and the results indicated that the downregulation of VEGF by ATRA was not Hif- $1 \alpha$-dependent, which is in contrast to most solid tumors (Fig. 3C). 
A
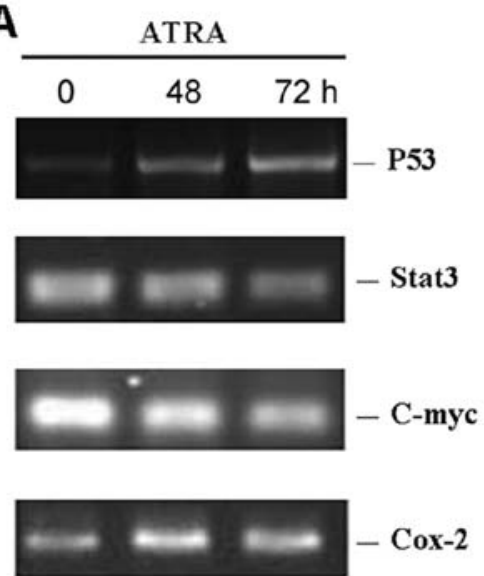
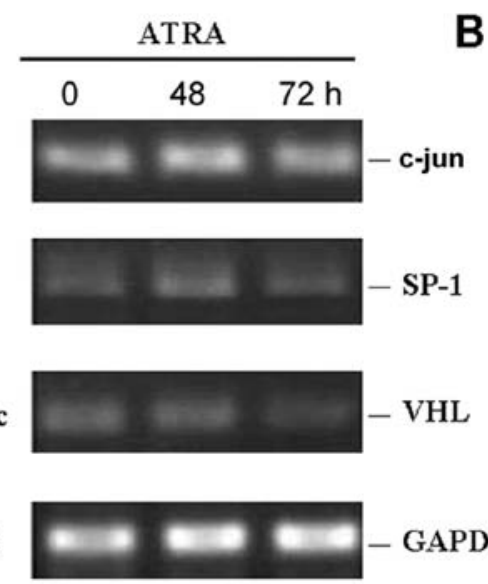

GAPDH

B
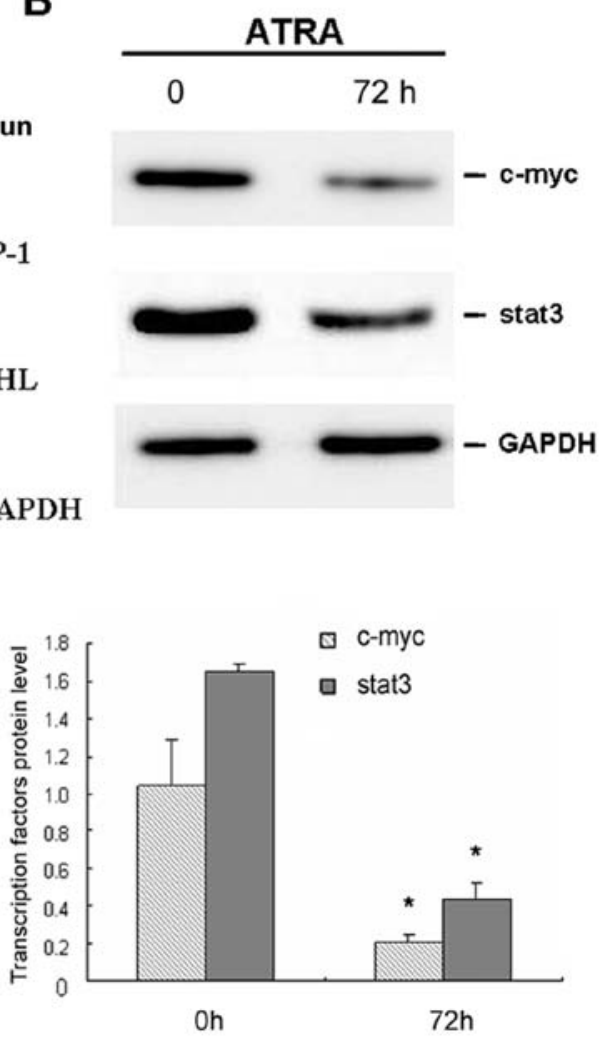

Figure 4. Effect of ATRA on transcription factors correlates with VEGF in HL-60 cells. (A) Expression of P53, SP-1, c-jun, VHL, cox-2, c-myc and stat3 in HL-60 cells was analyzed by RT-PCR following exposure to ATRA for 48 and $72 \mathrm{~h}$. Only c-myc and stat 3 were decreased, other factors were either enhanced or did not present any significant change. (B) Expression of c-myc and stat 3 protein was also downregulated by western blot analysis. "P<0.05.
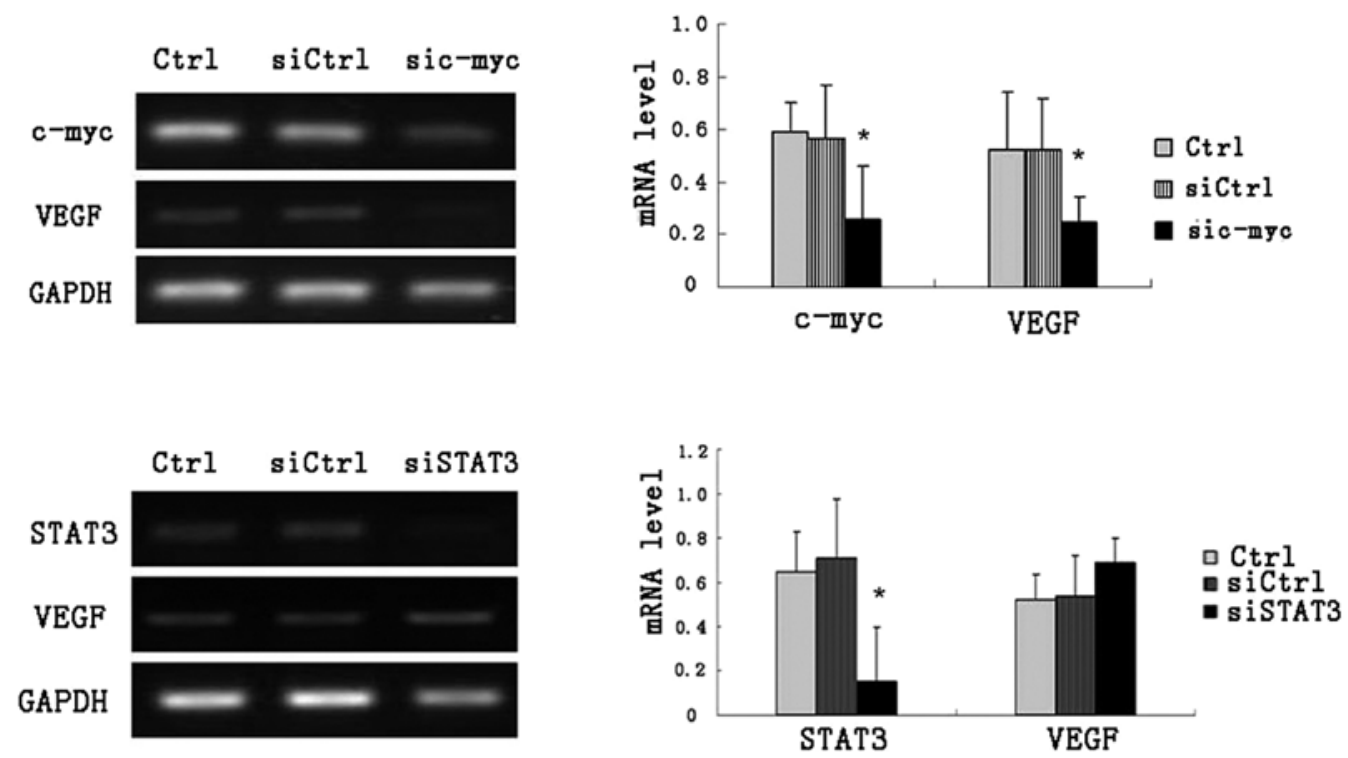

Figure 5. Expression of c-myc correlates with VEGF level in HL-60 cells. HL-60 cells were transfected with c-myc/stat3 siRNA, along with the AllStars negative control siRNA as negative control. VEGF expression was decreased in the sic-myc group. The data are presented as the means \pm SE. "P<0.05 vs. control.

c-myc correlates with the expression of VEGF in HL-60 cells treated with ATRA. Similar to the mechanism of VEGF observed in solid tumors, we selected seven transcription factors related to VEGF and performed RT-PCR to screening factors that may account for VEGF in HL-60 cells. As demonstrated in Fig. 4A, only stat3 and c-myc were significantly downregulated by ATRA, which was in agreement with VEGF expression following exposure to ATRA. Thus, stat3 and c-myc were selected for further confirmation by western blot assay (Fig. 4B). Consistent with the RT-PCR data, the expression levels of stat 3 and c-myc in HL-60 cells were both decreased following treatment with ATRA for $72 \mathrm{~h}$. 


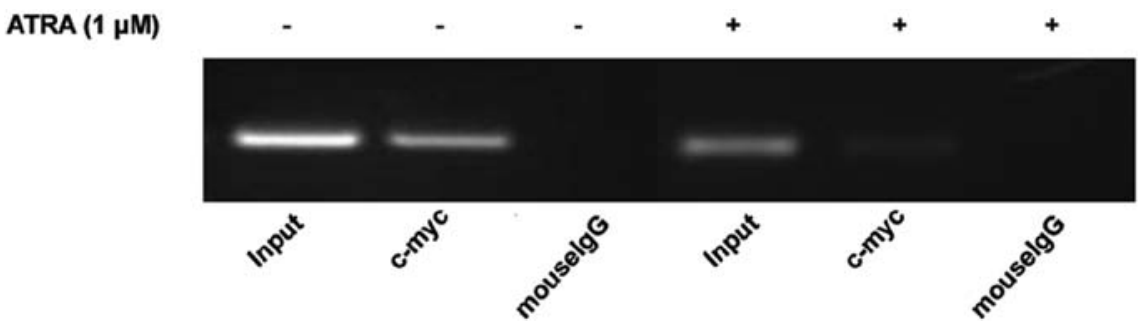

Figure 6. Effect of ATRA on c-myc binding on the VEGF promoter in HL-60 cells. HL-60 cells were treated with or without ATRA for 72 h. The immunoprecipitated DNA with rabbit normal IgG, c-myc antibody was amplified by PCR analysis for VEGF promoter.

Based on these results, specific siRNAs targeting stat 3 or $\mathrm{c}$-myc were investigated regarding their roles in regulating VEGF in HL-60 cells. The RT-PCR results showed that VEGF level could be downregulated significantly by silencing c-myc expression, other than stat3 siRNA (Fig. 5).

Effect of ATRA on the binding of c-myc to VEGF promoter in $H L-60$ cells. Recent studies revealed that c-myc activation is directly linked to the transcriptional regulation of VEGF by binding to the VEGF promoter. Based on this, we conducted ChIP assay. c-myc binding to VEGF promoter was detected (Fig. 6). As expected, ATRA treatment suppressed the binding of c-myc to VEGF promoter. These data further support the hypothesis that by downregulating the expression of c-myc, ATRA could inhibit mRNA and protein expression of VEGF, which may further influence the proliferation and differentiation of HL-60 cells.

\section{Discussion}

AML is a disease with a poor outcome, and the overall survival rate of five years is moderate to poor depending on age and cytogenetics (10). In 1985, ATRA was introduced to APL treatment. Optimization of the ATRA-based regimens combining ATRA and chemotherapy has further raised the complete remission (CR) rate up to $90-95 \%$, and a 6-year DFS up to $86 \%$ $( \pm 10 \%)$ in low-risk patients (11). At diagnosis, an enhanced microvessel density (MVD) in bone marrow biopsies has been observed, which is restored to normal levels when a CR has been achieved. Furthermore, AML bone marrow biopsies display enhanced angiogenesis and increased VEGF expression. The enhanced bone marrow vascularization is correlated with an increased expression of VEGF (10). However, the specific contribution of VEGF to the liquid tumor progression has not been clearly defined. Several groups have demonstrated that ATRA strongly induces terminal differentiation of HL-60 cells and reported ATRA treatment could downregulate VEGF significantly (12). Although VEGF is capable of signaling through VEGFR and induces endothelial proliferation and migration, the relationship between VEGF and the differentiative or proliferative ability of HL-60 remains unclear. In this study, by applying siRNA especially targeting VEGF, we demonstrated that HL-60 has a more invasive tumor phenotype inhibiting differentiation and triggering abnormal proliferation, all of which suggested that VEGF may participate in other leukemia-related pathways aside from the fact that it also plays a critical role in the growth of hematological neoplasm via autocrine or paracrine mechanism (13).
To further study the molecular mechanism regulating VEGF, we selected HIF-1 $\alpha$ as a cut-point, which appears to be a major regulator of VEGF gene expression in solid tumors $(14,15)$. Furthermore, this basic helix-loop-helix transcription factor may not only contribute to hypoxia-induced VEGF production, but it may also play a critical role in the oncogene-dependent expression of VEGF. However, in contrast to the previous results of solid tumors, our study found that the regulatory mechanism of VEGF in HL-60 cells induced by ATRA is independent of the HIF- $1 \alpha$-related pathway (6). These facts support the hypothesis that there do exist some mechanisms regulating VEGF other than HIF-1 $\alpha$. Through detailed analysis of the VEGF promoter and its regulatory transcription factors, previous studies have demonstrated some other critical factors, such as SP-1, c-jun, cox-2, c-myc and stat 3 , may be account for, which could also be disturbed under tumor microenvironment (16-20). These highlight the complex regulatory network of VEGF. Similar to the mechanism of VEGF observed in solid tumors, in the present study, we selected transcription factors concerning VEGF, such as P53, SP-1, c-jun, von Hippel-Lindau syndrome (VHL), cox-2, c-myc and stat 3 , to screen factors that may be directly correlated with VEGF in HL-60 cells. Following ATRA treatment, the expression of c-myc and stat 3 showed the same tendency as VEGF, which may indicate that other pathways account for abnormal VEGF expression. In this regard, we further investigated the roles of stat 3 and c-myc in regulating VEGF using specific siRNAs silencing these two factors in HL-60 cells separately, and the results indicated that inhibition of c-myc expression could downregulate VEGF, while the effect of stat3 on VEGF was not obvious, which was not consistent with the evidence in solid tumors. In summary, our results suggested the c-myc may be the upstream regulatory factor of VEGF.

In a previous study, Dadiani et al (21) found that, in breast cancer, both c-myc and the activated estrogen receptor $\alpha$ were shown to co-bind the VEGF promoter in close proximity, indicating a cooperative role for them in estrogen regulation of VEGF and the ability of c-myc to partially mimic estrogen regulation of angiogenesis. Mizukami et al (20) suggested that VEGF may also be an important target of c-myc in colon cancer, particularly under hypoxic conditions. In our study, we confirmed that c-myc could bind to VEGF promoter directly in HL-60 cells by ChIP, and this interaction may be markedly inhibited by ATRA, which decreased the expression of c-myc. Furthermore, Yang et al (22) co-delivered the pooled siRNAs targeting HDM2, c-myc and VEGF and it could effectively and simultaneously knock down their expressions and significantly inhibit tumor cell growth in A549 and H460 
cells in vitro. It is possible to establish other pooled siRNAs to inhibit the abnormal proliferation and promote the differentiation of HL-60 cells.

Collectively, our data demonstrated that in HL-60 human leukemia cells, VEGF play an important role in differentiation and proliferation, and ATRA promotes differentiation and inhibits proliferation by suppressing VEGF. c-myc, but not Hif-1 $\alpha$-dependent downregulation of VEGF, induced by ATRA contributes to the differentiation of HL-60 cells.

\section{Acknowledgements}

This study was supported by the Natural Science Foundation of China (30771103, 81172792), the Science and Technology Development Project of Shandong Province (2006GG2302010, 2007GG2002023) and the Natural Science Foundation of Shandong Province (Y2008C165, ZR2011HL050).

\section{References}

1. Olsson AK, Dimberg A, Kreuger J and Claesson-Welsh L: VEGF receptor signaling - in control of vascular function. Nat Rev Mol Cell Biol 7: 359-371, 2006

2. Podar K and Anderson KC: The pathophysiologic role of VEGF in hematologic malignancies: therapeutic implications. Blood 105: 1383-1395, 2005.

3. Mayerhofer M, Valent P, Sperr WR, Griffin JD and Sillaber C: $\mathrm{BCR} / \mathrm{ABL}$ induces expression of vascular endothelial growth factor and its transcriptional activator, hypoxia inducible factor-1alpha, through a pathway involving phosphoinositide 3-kinase and the mammalian target of rapamycin. Blood 100: 3767-3775, 2002.

4. Perez-Atayde AR, Sallan SE, Tedrow U, Connors S, Allred E and Folkman J: Spectrum of tumor angiogenesis in the bone marrow of children with acute lymphoblastic leukemia. Am J Pathol 150: 815-821, 1997.

5. Ghosh AK, Shanafelt TD, Cimmino A, et al: Aberrant regulation of pVHL levels by microRNA promotes the HIF/VEGF axis in CLL B cells. Blood 113: 5568-5574, 2009.

6. Jiang GS, Yang WH, Wen PE, Ren X, Tang TH and Ren HQ: HIF-1 $\alpha$ independent down-regulation of VEGF expression in HL60 cells after in vitro exposure to ATRA. Blood 110: 4290, 2007.

7. Lidgren A, Hedberg Y, Grankvist K, Torgny R, Vasko J and Ljungberg B: The expression of hypoxia-inducible factor lalpha is a favorable independent prognostic factor in renal cell carcinoma. Clin Cancer Res 11: 1129-1135, 2005.

8. Schuch G, Machluf M, Bartsch G Jr, et al: In vivo administration of vascular endothelial growth factor (VEGF) and its antagonist, soluble neuropilin-1, predicts a role of VEGF in the progression of acute myeloid leukemia in vivo. Blood 100: 4622-4628, 2002.
9. Ter Elst A, Ma B, Scherpen FJ, et al: Repression of vascular endothelial growth factor expression by the runt-related transcription factor 1 in acute myeloid leukemia. Cancer Res 71: 2761-2771, 2011.

10. Weidenaar AC, ter Elst A, Koopmans-Klein G, et al: High acute myeloid leukemia derived VEGFA levels are associated with a specific vascular morphology in the leukemic bone marrow. Cell Oncol 34: 289-296, 2011.

11. Bajpai J, Sharma A, Kumar L, et al: Acute promyelocytic leukemia: an experience from a tertiary care centre in north India. Indian J Cancer 48: 316-322, 2011.

12. Tee MK, Vigne JL and Taylor RN: All-trans retinoic acid inhibits vascular endothelial growth factor expression in a cell model of neutrophil activation. Endocrinology 147: 1264-1270, 2006.

13. Wang ES, Teruya-Feldstein J, Wu Y, Zhu Z, Hicklin DJ and Moore MA: Targeting autocrine and paracrine VEGF receptor pathways inhibits human lymphoma xenografts in vivo. Blood 104: 2893-2902, 2004

14. Vaupel P: The role of hypoxia-induced factors in tumor progression. Oncologist 9 (Suppl 5): 10-17, 2004.

15. Ohno H, Shirato K, Sakurai T, et al: Effect of exercise on HIF-1 and VEGF signaling. J Phys Fitness Sports Med 1: 5-16, 2012.

16. Loeffler S, Fayard B, Weis J and Weissenberger J: Interleukin-6 induces transcriptional activation of vascular endothelial growth factor (VEGF) in astrocytes in vivo and regulates VEGF promoter activity in glioblastoma cells via direct interaction between STAT3 and Sp1. Int J Cancer 115: 202-213, 2005.

17. Xie K, Wei D, Shi Q and Huang S: Constitutive and inducible expression and regulation of vascular endothelial growth factor. Cytokine Growth Factor Rev 15: 297-324, 2004.

18. Colla S, Tagliaferri S, Morandi F, et al: The new tumor-suppressor gene inhibitor of growth family member 4 (ING4) regulates the production of proangiogenic molecules by myeloma cells and suppresses hypoxia-inducible factor-1 alpha (HIF-1alpha) activity: involvement in myeloma-induced angiogenesis. Blood 110: 4464-4475, 2007.

19. Lee CC, Chen SC, Tsai SC, et al: Hyperbaric oxygen induces VEGF expression through ERK, JNK and c-Jun/AP-1 activation in human umbilical vein endothelial cells. J Biomed Sci 13: 143-156, 2006.

20. Mizukami Y, Fujiki K, Duerr EM, et al: Hypoxic regulation of vascular endothelial growth factor through the induction of phosphatidylinositol 3-kinase/Rho/ROCK and c-Myc. J Biol Chem 281: 13957-13963, 2006.

21. Dadiani M, Seger D, Kreizman T, et al: Estrogen regulation of vascular endothelial growth factor in breast cancer in vitro and in vivo: the role of estrogen receptor alpha and c-Myc. Endocr Relat Cancer 16: 819-834, 2009.

22. Yang Y, Hu Y, Wang Y, Li J, Liu F and Huang L: Nanoparticle delivery of pooled siRNA for effective treatment of non-small cell lung cancer. Mol Pharm: Jun 22, 2012 (Epub ahead of print). 